Published in final edited form as:

Langmuir. 2019 September 17; 35(37): 12071-12078. doi:10.1021/acs.langmuir.9b01288.

\title{
Lipid and Protein Transfer between Nanolipoprotein Particles and Supported Lipid Bilayers
}

\author{
Amanda T. Dang ${ }^{\dagger}$, Wei He ${ }^{\ddagger}$, Daniela B. Ivey ${ }^{\#}$, Matthew A. Coleman ${ }^{\ddagger}$, Tonya L. Kuhl ${ }^{\star}, \#$ \\ tDepartment of Materials Science and Engineering, University of California, Davis CA 95616 \\ ‡Lawrence Livermore National Laboratory, Livermore, CA 94550 \\ \#Department of Chemical Engineering, University of California, Davis CA 95616
}

\begin{abstract}
A nanolipoprotein particle (NLP) is a lipid bilayer disc stabilized by two amphipathic "scaffold" apolipoproteins. It has been most notably utilized as a tool for solubilizing a variety of membrane proteins while preserving structural and functional properties. Transfer of functional proteins from NLPs into model membrane systems such as supported lipid bilayers (SLBs) would enable new opportunities for example: two-dimensional protein crystallization and studies on protein-protein interactions. This work used fluorescence microscopy (FM) and atomic force microscopy (AFM) to investigate the interaction between NLPs and SLBs. When incubated with SLBs, NLPs were found to spontaneously deliver lipid and protein cargo. The impact of membrane composition on lipid exchange was explored, revealing a positive correlation between the magnitude of lipid transfer and concentration of defects in the target SLB. Incorporation of lipids capable of binding specifically to polyhistidine tags encoded into the apolipoproteins also boosted transfer of NLP cargo. Optimal conditions for lipid and protein delivery from NLPs to SLBs are proposed based on interaction mechanisms.
\end{abstract}

\section{Graphical Abstract}

"Corresponding Author: tlkuhl@ucdavis.edu.

Author Contributions

The manuscript was written through contributions of all authors. All authors have given approval to the final version of the manuscript.

Detailed methods, sample specifications, and additional figures in Supporting Information. 


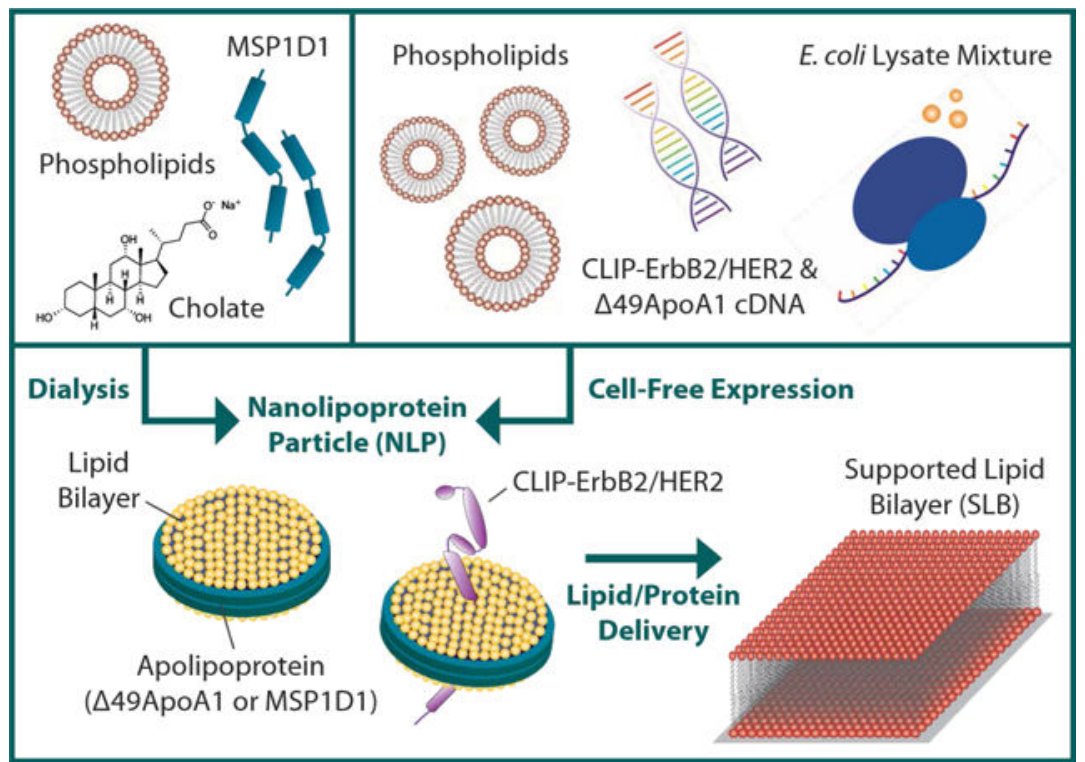

Schematic of NLP assembly methods and delivery of lipids/proteins from NLPs to SLBs.

\section{Keywords}

nanolipoprotein particle; supported lipid bilayer; atomic force microscopy; apolipoprotein; membrane scaffold protein

\section{INTRODUCTION}

Over the past two decades, the biomimetic nanolipoprotein particle (NLP) a.k.a. nanodisc ${ }^{1}$ or reconstituted high-density lipoprotein (HDL) particle, has been steadily developed as a promising drug delivery vehicle ${ }^{2-3}$ as well as a proficient tool for the expression and characterization of assorted membrane proteins. ${ }^{4-6}$ Biocompatible and amenable to modification for enhanced serum stability, ${ }^{7}$ NLPs emulate naturally occurring HDL particles, which transport lipidic cargo in the circulatory system. ${ }^{8-9}$ HDL particles are soluble lipoprotein complexes with cores comprised of triglycerides, cholesterol esters, and cholesterols, encapsulated by a phospholipid bilayer and secured by amphipathic apolipoproteins. ${ }^{10}$ Apolipoproteins mediate interactions with plasma membrane surface receptors and enable delivery of cholesterol via selective cellular uptake pathways. ${ }^{8,}$ 11-12 Additionally, they act as "scaffold" proteins that control particle size and structure.

In distinction from their biological HDL counterparts, ApoA1-based NLPs are discoidal lipid bilayers with an average thickness of $5 \mathrm{~nm}$ and diameters that range between $10-30$ nm depending on the specific apolipoprotein and its ratio compared to lipids. ${ }^{4,}{ }^{13-15}$ Each ApoA1-based NLP has two apolipoproteins that insulate the hydrophobic core and dictate particle size. There are two popular methods for assembling NLPs in vitro. The first is reconstitution of apolipoprotein in the presence of lipids (vesicles) and detergent, ${ }^{1,16-17}$ where gradual dilution of the detergent induces self-assembly of NLPs. The second is cellfree expression of apolipoprotein in the presence of lipids. Importantly, the NLP assembly 
techniques result in production of identical nanoparticles. ${ }^{14}$ Since the apolipoprotein can be made to encode a polyhistidine tag, NLPs can be purified using straightforward affinity chromatography procedures. The principle advantage of cell-free expression is its capacity for simultaneous production of apolipoproteins and membrane proteins without the need for adding or removing detergents. ${ }^{4}$ When co-expressed with NLPs, membrane proteins have been found to insert into NLP bilayers to form a stably solubilized membrane protein-NLP complexes (MP-NLPs). Importantly, membrane proteins in MP-NLP complexes have been shown to retain their structure and functionality using the "One-Pot" cell-free approach. $.5,18$

MP-NLP complexes have potential for broad utility in pharmaceuticals applications and in fundamental research on protein-protein and protein-lipid interactions. While strategies for preparation and characterization of proteins using high spatial resolution analytical techniques such as electron microscopy and X-ray crystallography have steadily improved, the crucial task of examining protein structure and functionality in native lipid environments remains a persistent challenge. ${ }^{19}$ Even though membrane proteins make up over $60 \%$ of drug targets ${ }^{20}$ less than $1 \%$ of solved protein crystal structures are membrane proteins. ${ }^{21}$ This is compelling motivation for development of broadly applicable, lipid-based characterization platforms, especially amid growing evidence that lipids not only stabilize, ${ }^{22}$ but regulate membrane protein activity. ${ }^{23-24}$ MP-NLPs offer one modality for examining properties of membrane proteins embedded in lipid bilayers. Several studies using NLPs to examine protein activity, ${ }^{25}$ oligomerization, ${ }^{26-27}$ and diffusion dynamics ${ }^{18,} 28$ have been reported.

Another platform that recapitulates physicochemical properties of native membranes is the supported lipid bilayer (SLB). Similar to NLPs, SLBs are powerful tools for studying membrane proteins because they allow for observation of protein-lipid interactions, ${ }^{24}, 29$ protein-protein interactions, ${ }^{30}$ conformational changes, and hydrophobic mismatch with high fidelity. ${ }^{31}$ A SLB is a lipid bilayer that has been reconstituted on a solid, normally planar substrate such as silica or mica. ${ }^{32}$ SLBs are generally more robust than freestanding bilayers. Further, SLBs are compatible with two-dimensional characterization methods including wide-field fluorescence microscopy (FM), electron microscopy, atomic force microscopy (AFM), and neutron or X-ray scattering. In addition, SLB lipid composition can be precisely tailored to simulate nano- to microscopic phase separation and lipid raft domains. ${ }^{33}$ For studies on membrane proteins, SLBs can be modified with polymer cushions $^{34-36}$ to prevent unwanted interactions between embedded proteins and the underlying substrate. SLBs can also be micropatterned ${ }^{37}$ to manipulate the spatial organization of associated molecules.

Mechanisms by which NLPs transfer lipids and proteins to and from other lipid constructs have been explored with bicelles ${ }^{27,38}$ and with other NLPs, ${ }^{39}$ but scarcely with continuous bilayers. A recent study by Patriarchi et al. ${ }^{40}$ showed that NLPs could deliver functional $\beta_{2}-$ adrenergic receptor, a G-protein coupled receptor (GPCR), into the plasma membrane of living cells. However, the underlying mechanism behind the protein transfer process remains obscure. In this work, we used wide-field FM and AFM to determine the impacts of composition and the presence of defects on lipid cargo transfer from NLPs to SLBs. 


\section{MATERIALS AND METHODS}

\section{General Materials and Methods}

MilliQ deionized water (resistivity $\geq 18 \mathrm{M} \Omega \cdot \mathrm{cm}$ ) from a Barnstead water purification system (Thermo Fisher Scientific) was used in all protocols except cell-free expression, which utilized DNA-ase free water included in the Invitrogen Expressway Maxi kit. The lipids 1,2dimyristoleoyl-sn-glycero-3-phosphocholine (DMPC), 1,2-dipalmitoyl-sn-glycerol-3Phosphatidylcholine (DPPC), 1,2-dipalmitoylsn-glycero-3-phosphoethanolamine (DPPE), 1,2-dioleoyl-sn-glycero-3-phosphocholine (DOPC), 1,2-dioleoyl-sn-glycero-3-[(N-(5amino-1-carboxypentyl)iminodiacetic acid)succinyl] (nickel salt) (DOGS-NTA-Ni), and 1,2dipalmitoyl-sn-glycero-3-phosphoethanolamine-N-(lissamine rhodamine B sulfonyl) (ammonium salt) (Rhodamine-DHPE) were purchased from Avanti Polar Lipids, Inc. Texas Red $^{\mathrm{TM}}$ 1,2-Dihexadecanoyl-sn-Glycero-3-Phosphoethanolamine, Triethylammonium Salt (TR-DHPE) and Oregon Green ${ }^{\mathrm{TM}} 488$ 1,2-dihexadecanoyl-sn-glycero-3phosphoethanolamine (OG-DHPE) labeled lipid reagents were purchased from Thermo Fisher Scientific. Lipids were dissolved in Chloroform, HPLC grade (Thermo Fisher Scientific) to make solutions at a concentration of $1 \mathrm{mg} / \mathrm{ml}$. Sodium chloride ( $\geq 99 \%$ purity), imidazole ( $\geq 99 \%$ purity), Trizma ${ }^{\circledR}$ hydrochloride and Trizma ${ }^{\circledR}$ base were used to prepare pH 7.4 Tris buffer. Sodium cholate hydrate ( $\geq 99 \%$ purity) was purchased from SigmaAldrich, Inc. Phosphate buffered saline (PBS) dry pack, ultra-pure grade, was purchased from Apex Bioresearch, Inc. and dissolved in MilliQ deionized water to make PBS buffer (10 mM phosphate buffer, $137 \mathrm{mM} \mathrm{NaCl}, 2.7 \mathrm{mM} \mathrm{KCl}$ ).

Glass coverslips (Thermo Fisher Scientific) were cleaned with Hellmanex basic detergent (Sigma-Aldrich), rinsed with MilliQ deionized water followed by 200 proof ethanol, and dried under a stream of nitrogen (specialty grade, $99.998 \%$ pure). Lastly, glass substrates were UV-ozone treated to promote hydroxyl group formation at the surface and used within 30 min of treatment. Coverslips functionalized with poly(acrylic acid) (PAA) cushions were additionally subjected to sonication in acetone, then isopropyl alcohol to ensure full removal of organic contaminants from the surface prior to UV-ozone treatment and PAA deposition. Mica (Axim Mica) was freshly cleaved before use.

\section{PAA Cushion Preparation}

PAA cushions were prepared using spin-coating methods previously described by El-Khouri et al. ${ }^{34}$ Basically, deposition solutions were prepared by dissolving PAA (450k MW, 0.1\% cross-linked, Sigma-Aldrich, Inc.) in methanol ( $\geq 99 \%$ purity, Sigma-Aldrich) at a concentration of $1 \mathrm{mg} / \mathrm{mL}$. To enable covalent grafting of polymer chains to the silica substrate, coverslip surfaces were initially functionalized with aminopropyltriethoxysilane (APTES, Gelest, Inc.) by solution deposition in toluene ( $299 \%$ purity, Sigma-Aldrich). The PAA cushion was then formed by spin-coating and subsequent curing. Finally, PAA substrates were immersed in Tris buffer ( $\mathrm{pH} 9$ ) to convert anhydrides to carboxylates and relieve mechanical stresses in the polymer layer. As previously reported, ${ }^{34}$ cushion thickness can be modified by altering the concentration of the PAA in the spin-coating solution. The technique of UV-Ozone photolithography was used to pattern PAA cushions into arrays of $100-200 \mu \mathrm{m}$ squares. Areas exposed during photolithography were subsequently treated 
with AquaSil siliconizing fluid (Thermo Fisher Scientific) to render them resistant to nonspecific protein adsorption. Additional details for PAA-cushion preparation is provided in the Supporting Information.

\section{SLB Sample Preparation}

SLBs were deposited on bare glass coverslips (Thermo Fisher Scientific) or freshly cleaved mica (Axim Mica) using vesicle fusion, Langmuir-Blodgett (LB)-LB deposition, or LBLangmuir-Schaeffer (LS) deposition. In LB-LB deposition, each leaflet was added by moving the substrate vertically through a compressed lipid monolayer at an air-water interface. In LB-LS deposition, the outer leaflet was transferred by lowering the substrate through the air-water interface oriented parallel to the monolayer. ${ }^{32,41}$ Details on methods used to produce each SLB are summarized in Table S1 in Supporting Information.

\section{Vesicle Sample Preparation}

Vesicle solutions for comparative lipid exchange experiments were prepared by sonication. A mixture of DMPC containing 2 mol\% Rhodamine-DHPE was dissolved in chloroform, dried under nitrogen, and placed under vacuum for at least $4 \mathrm{~h}$. The mixture was hydrated with PBS buffer to a concentration of $0.1 \mathrm{mg} / \mathrm{mL}$, vortexed for $15 \mathrm{sec}$, and then placed in an ultrasonic bath sonicator for $30 \mathrm{~min}$. Vesicle solutions were always used within 1 hour after preparation. Size determination by dynamic light scattering (DLS, Malvern Zeta Sizer) revealed an average vesicle size of $55.5 \pm 11 \mathrm{~nm}$ in diameter.

\section{NLP Assembly from Lyophilized Apolipoprotein}

The NLPs stabilized with membrane scaffold protein MSP1D1 (MSP1D1-NLPs) were assembled using methods adopted from the process described by Zeno et al. ${ }^{42}$ In brief, a stoichiometric excess of DMPC doped with 2 mol\% Rhodamine-DHPE was dried in a glass vial with nitrogen and placed under mild vacuum for at least $6 \mathrm{hr}$. The lipid mixture was then hydrated in Tris buffer containing sodium cholate hydrate and added to another aliquot of Tris buffer containing MSP1D1. Each MSP1D1 had a chain length of 217 amino acids (25.3 $\mathrm{kDa}$ ) and a polyhistidine tag attached to its $\mathrm{N}$-terminus. After incubation, the mixture was transferred to a $10 \mathrm{kDa}$ MWCO Slide-A-Lyzer dialysis cassette (Thermo Fisher Scientific) and dialyzed at $4{ }^{\circ} \mathrm{C}$, in Tris buffer ( $\mathrm{pH}$ 7.4) to promote cholate removal and NLP assembly. Subsequent purification (described below) ensured complete cholate removal from the final product. For the detailed protocol for synthesis of MSP1D1-NLPs, see Supporting Information.

\section{NLP Assembly using Cell-Free Expression}

The NLPs stabilized with apolipoprotein $\triangle 49$ ApoA1 ( $\triangle 49$ ApoA1-NLPs) and with embedded receptor tyrosine kinase CLIP-ErbB2/HER2 (CLIP-ErbB2-NLPs) were prepared using cell-free expression methods described by He et al. ${ }^{5}$ The cell-free reaction was carried out using the Invitrogen Expressway ${ }^{\mathrm{TM}}$ Maxi Cell-Free E. coli Expression System (Thermo Fisher Scientific). Plasmids encoding CLIP-ErbB2/HER2 and a truncated 6xHis-tagged version of human apolipoprotein A-I, missing the amino-terminal 49 amino acids ( $\triangle 49$ ApoA1) were codon optimized for $E$. coli expression. ${ }^{5} \Delta 49$ ApoA1-NLPs were 
assembled with DMPC vesicles containing 0.5 mol\% TR-DHPE. CLIP-ErbB2 NLPs were conjugated with fluorescent substrate CLIP-Cell TMR-Star (New England Biolabs), added to the cell-free reaction $1 \mathrm{hr}$ prior to purification.

\section{NLP Purification and Verification}

All NLPs were isolated by nickel affinity purification. Initially, samples were incubated for 1 hr with HisPur ${ }^{\mathrm{TM}}$ Ni-NTA resin (Thermo Fisher Scientific), rinsed with Tris buffer (pH 7.4) at $4^{\circ} \mathrm{C}$. Next, 3-4 wash steps were then performed using Tris buffer containing $20 \mathrm{mM}$ imidazole, followed by 3-4 elution steps with Tris buffer containing $400 \mathrm{mM}$ imidazole. NLPs in the eluted fraction were concentrated using a Vivaspin $100 \mathrm{kDa}$ MWCO centrifugal concentrator $^{13}$ (Thermo Fisher Scientific). To remove imidazole from the solution, concentrated samples were transferred to $10 \mathrm{kDa}$ MWCO Slide-A-Lyzer dialysis cassettes (Thermo Fisher Scientific) and dialyzed at $4{ }^{\circ} \mathrm{C}$, in PBS buffer (pH 7.4) at over 500 times the sample volume ( 4 buffer exchanges over 36 hours). Recovered samples were stored in the dark at $4{ }^{\circ} \mathrm{C}$ until use.

Production of MSP1D1, $\triangle 49$ ApoA1, and CLIP-ErbB2/HER2 protein particles was verified by SDS-PAGE. Protein concentration in each sample was adjusted to $0.1 \mathrm{mg} / \mathrm{mL}$ and verified by measuring peak absorbance at $280 \mathrm{~nm}$ using a UV-vis spectrophotometer (Shimadzu Scientific Instruments). The average discoidal diameter of MSP1D1-NLPs, determined by DLS (Malvern Zeta Sizer), was $20.7 \pm 1.6 \mathrm{~nm}$ and comparable to sizes reported for $\triangle 49$ ApoA1-NLPs ${ }^{4}$ and CLIP-ErbB2-NLPs. ${ }^{5}$ Additional details on NLP verification are included in the Supporting Information.

\section{NLP Incubation with SLBs}

At the start of each incubation step, $50 \mu \mathrm{L}$ of $0.1 \mathrm{mg} / \mathrm{mL}$ NLP solution was pipetted into a 3 $\mathrm{mL}$ volume of PBS buffer containing the SLB (final NLP concentration $\sim 1.6 \mu \mathrm{g} / \mathrm{mL}$ ). At the end of each incubation period, residual NLPs or vesicles were removed by exchanging the incubation solution covering the SLB with fresh buffer. Total incubation time was varied to suit the unique conditions of each experiment. For example, in FM measurements, MSP1D1-NLPs and $\triangle 49$ ApoA1-NLPs were incubated with glass-supported bilayers for 15 min and PAA-cushioned SLBs for $30 \mathrm{~min}$, respectively. However, CLIP-ErbB2-NLPs were incubated for $12 \mathrm{hr}$ with PAA-cushioned SLBs because earlier trials (unpublished data) indicated that at least several hours were required to accumulate enough protein in the SLB to produce an appreciable fluorescent signal. For lengthier AFM experiments, $\triangle 49 \mathrm{ApoA} 1-$ NLPs were also incubated for $12 \mathrm{hr}$ with SLBs. The extended duration was necessary to assure the samples had reached static equilibrium before the start of topography scans.

\section{Fluorescence Microscopy}

The FM images were acquired using a Nikon Eclipse E600 microscope connected to an Andor Zyla sCMOS camera (DG-152V-C1E-FI) at 40X magnification. A mercury short-arc lamp (Osram, HBO 100W/2) was used to illuminate samples. A TRITC filter cube (dichroic cut-on wavelength $562 \mathrm{~nm}$ ), TR filter cube (dichroic cut-on wavelength $593 \mathrm{~nm}$ ), and FITC filter cube (dichroic cut-on wavelength $506 \mathrm{~nm}$ ) were used to isolate fluorescence emission from Rhodamine-DHPE/CLIP-Cell TMR-Star, TR-DHPE, and OG-DHPE, respectively. For 
mean fluorescence intensity measurements, at least 10 images were recorded across the surface of each sample and analyzed using a MATLAB program (see Supporting Information for details).

\section{Atomic Force Microscopy}

AFM images were acquired in PBS buffer using a MFP3D-SA system (Asylum Research, Santa Barbara, CA) equipped with a medium soft silicon cantilever (model AC240TS-R3, Asylum Research, Santa Barbara, CA) with a spring constant of $2 \mathrm{~N} / \mathrm{m}$. Images were recorded in tapping mode AFM with a set point of $250-300 \mathrm{mV}$ and a scan rate of $1.5 \mathrm{~Hz}$. AFM images were analyzed using Gwyddion ver. 2.49.

\section{RESULTS}

\section{Impact of SLB Composition on Lipid Exchange}

Figure 1 shows the mean fluorescence intensity of various composition SLBs after incubation with fluorescently labeled MSP1D1-NLPs and fluorescently labeled control vesicles, normalized by the fluorescence intensity of unlabeled SLBs on glass. Combined with qualitative observations (Figure 2), these results provide insight into the influence of target membrane composition and properties for optimizing NLP-SLB interactions and material transfer from NLPs to the SLB. Lipid transfer was markedly enhanced by defects in the supported membrane and prevented in well-packed, gel-phase membranes. The SLB with the most pronounced lipid exchange with NLPs had an outer leaflet composition of DMPC + 5 mol\% DOGS-NTA and an inner leaflet composition of DMPC. DMPC membranes are near the phase-transition state at room temperature $\left(\mathrm{T}_{\mathrm{m}}=24{ }^{\circ} \mathrm{C}\right)$. Lipid transfer was further enhanced by doping the SLB mixture with DOGS-NTA. DOGS-NTA, a nickel-chelating lipid, is capable of specific binding to the polyhistidine-tag of the apolipoprotein, thus acting as an anchor point. When SLBs comprised of DMPC $+5 \mathrm{~mol} \%$ DOGS-NTA were supported by gel phase inner leaflets comprised of DPPE $\left(\mathrm{T}_{\mathrm{m}}=63{ }^{\circ} \mathrm{C}\right)$, the concentration of defects was reduced ${ }^{32,43}$ and a corresponding reduction in lipid exchange was observed.

In fluid phase membranes comprised of DOPC $+5 \mathrm{~mol} \%$ DOGS-NTA $\left(\mathrm{T}_{\mathrm{m}}=-17^{\circ} \mathrm{C}\right)$, where defects were relatively abundant, lipid exchange was significant and comparable to the amount observed in DMPC +5 mol\% DOGS-NTA. Phase separation in these otherwise homogeneous samples signaled that both DMPC and fluorescent lipid Rhodamine B-DHPE were delivered. In phase separated SLBs comprised of an inner DPPC $\left(\mathrm{T}_{\mathrm{m}}=41^{\circ} \mathrm{C}\right)$ leaflet and an outer DPPE:DOPC (3:7 molar ratio) leaflet, the prevalent fluid phase meant that lipid exchange was only slightly suppressed despite having a gel phase inner leaflet and a fraction of the surface occupied by gel phase domains. Interestingly, incorporation of DOGS-NTA into DPPE:DOPC membranes did not boost lipid exchange the same way it did for DMPC membranes. Instead, incorporation of DOGS-NTA into the phase separated systems reduced the standard deviation across samples, perhaps by driving broader incorporation of fluorescent lipids across the SLB surface as opposed to localized exchange with defects. Homogeneous gel-phase SLBs showed no evidence of lipid exchange upon extended incubation with NLPs. 
There was more lipid exchange from NLPs compared to vesicle controls (Figure 2 insets). When defects were suppressed in DMPC + 5 mol\% DOGS-NTA SLBs with inner leaflets comprised of DPPE, similar levels of materials transfer to the SLB were obtained.

Fluorescence recovery after photobleaching (FRAP), an indicator of the lateral mobility of fluorescently labeled lipids in the SLB, was consistently observed in samples incubated with NLPs (see Supporting Information Figure S3). Furthermore, the intensity measurements of SLBs incubated with vesicles were often artificially enhanced by the presence of adsorbed vesicles on the surface (see Supporting Information Figure S4). Taken together, these results demonstrate that NLPs offer an improvement over vesicle incubation and are a viable delivery vehicle for transferring small biomolecules such as lipids to a variety of SLB compositions. Exchange is optimized in the presence of defects or, when defects are suppressed, the presence of specific, NLP binding lipids at the SLB surface.

\section{AFM of SLBs Incubated with NLPs}

Topographic images acquired by AFM suggest a highly complex mechanism of interaction between NLPs and SLBs. Figure 3 shows AFM images of SLBs with outer and inner leaflets comprised of DMPC + 5 mol\% DOGS-NTA and DPPE, respectively, incubated with and without NLPs. Phase separation and defects in the form of transmembrane holes (depth $\sim 5$ $\mathrm{nm}$ ) are evident in the control sample. This microstructure is typical for DMPC membranes which reside in near-transition states at room temperature. In the sample incubated with NLPs, there is no discernable phase separation and holes are absent in the membrane. There are also ring-like features with an average height of $\sim 1 \mathrm{~nm}$ distributed across the surface. In previous attempts to image the interaction between NLPs and the SLB in situ, nonspecific adsorption of NLPs to the AFM probe disrupted image acquisition. Consequentially, it was difficult to determine the exact mechanism behind this complex surface rearrangement. However, the size distribution of the rings $(122.1 \pm 85 \mathrm{~nm})$ was comparable to that of defects in the control sample (143.2 $\pm 74 \mathrm{~nm}$ ), suggesting that defects act as docking sites for the NLPs and play a key a role in mediating the formation of the final nanostructure.

Figure 4 shows AFM images of SLBs with outer and inner leaflets comprised of DPPC:DOPC + 5 mol\% DOGS-NTA:Chol (9:9:2 molar ratio) and DPPE, respectively, incubated with and without NLPs. Phase separation and transmembrane holes were again observed in the control sample. Like the DMPC membrane results, incubating NLPs with DPPC:DOPC:Chol membranes appeared to result in suppressed phase separation. Line profile scans exposed defects with a similar size distribution $(501.2 \pm 334 \mathrm{~nm})$ to that of defects observed in the control sample $(456.7 \pm 234 \mathrm{~nm})$. However, defects in SLBs incubated with NLPs had a shallower depth of $\sim 1.5 \mathrm{~nm}$ compared to $\sim 5 \mathrm{~nm}$ in the control samples. Assuming that lipid transfer from the NLPs resulted in filling of defects in both of the compositions examined using AFM, increased lateral pressure from lipid crowding would induce mixing in the system and suppress phase separation as observed. ${ }^{39}$

\section{Lipid Transfer between NLPs and PAA-Cushioned SLBs}

Figure 5A shows FM images of a patterned PAA-cushioned membrane labeled with OGDHPE. As seen in Figure 5B, addition of $\triangle 49$ ApoA1-NLPs containing TR-DHPE resulted in lipid transfer, made apparent by co-localization of TR-DHPE and OG-DHPE emission on 
membrane patches. When the experiment was repeated with CLIP-ErbB2-NLPs (labeled with CLIP-Cell TMR-Star), the SLB was not labeled to reduce background noise. After a 12 $\mathrm{hr}$ incubation period, CLIP-ErbB2/HER2 transfer was confirmed when emission from the conjugated fluorescent substrate, CLIP-Cell TMR-Cell was detected in the SLB. Details on negative control trials are described in Supporting Information. FRAP experiments revealed that CLIP-ErbB2/HER2 exhibited lateral mobility, but only in areas where the membrane appeared to be suspended between PAA patches (Figure 6). Restricted mobility in directly supported membranes was likely due to the $\mathrm{pH}$-sensitive properties of PAA cushions. When stored in acidic conditions $(\mathrm{pH}<6)$ or in solutions with low ionic strength, the polymer assumes a collapsed structure where lateral diffusivity in the supported bilayer can be reduced by several orders of magnitude compared to a fluid bilayer supported on an oxide substrate. ${ }^{34}$ While not done in this experiment, it is possible to induce mobility in PAAcushioned SLBs by adjusting the $\mathrm{pH}$ to 7.4 or higher. Under neutral to alkaline conditions, coupling between the cushion and the lipid bilayer becomes weakened as ionization of PAA chains causes the former to swell with water. ${ }^{34}$ Note that effective $\mathrm{pH}$ equilibration requires that ions be able to diffuse into the water layer between the membrane and underlying substrate. However, a tightly packed, continuous bilayer may effectively "seal" the PAA cushion from the bulk solution. This issue could be circumvented by introducing defects or channels into the membrane. Straightforward methods for doing so include, patterning, thermal cycling, and incorporation of transmembrane channel proteins such as gramicidin. In general, pH-sensitive membranes such as PAA-cushioned SLBs offer a convenient mode of controlling the diffusivity of proteins in model systems through minor adjustments to solution $\mathrm{pH}$.

\section{Mechanisms of Transfer}

Enhanced transfer in samples with more defects and containing DOGS-NTA suggests that materials delivery from NLPs can be regulated by the target lipid environment and by the presence of apolipoprotein-binding molecules. These results are in accord with previous studies on lipid exchange between NLPs and other biomimetic lipid systems. ${ }^{38-39}$ Still, the vast parameter space around this complex interaction makes it difficult to define an exact mechanism. Given the scarcity of information pertaining to NLP-SLB systems, tangential research from HDL particles may provide clues on the range of behaviors that might be further explored. In biological systems, HDL particles are thought to deliver lipids directly to cell membranes upon binding with the scavenger receptor, class B type 1 (SR-B1). ${ }^{44-45}$ Importantly, lipid uptake is selective and does not compromise the structural fidelity of the HDL particle when mediated by SR-B $1 .{ }^{11}$ Yet in another independent study on model membranes, HDL particles appear to integrate upon contact even in the absence of surface receptors. ${ }^{8}$ Further, associated apolipoproteins do not appear to discriminate between different types of lipid domains. ${ }^{46}$ Unsurprisingly, NLPs exhibit similar capacity for cargo delivery through either specific or non-specific interactions with SLBs. In the case of the latter, defects appear to serve as the principle sites for materials exchange based on the results of this study. Continuing efforts to determine mechanisms of NLP-SLB interactions would benefit from examining the kinetics of lipid transfer and the residence time of apolipoprotein at the SLB surface, with and without specific binding receptor analogs. 


\section{CONCLUSIONS}

This work explored the viability of using NLPs to deliver lipids and proteins to SLBs. FM experiments revealed that the efficacy of lipid exchange depended on the composition of the target bilayer. Transfer was improved by incorporating polyhistidine tag-binding lipids into the SLB which selectively bound NLP apolipoproteins encoded with polyhistidine tags. The most dominant influence on the magnitude of lipid exchange appeared to be the concentration of defects in the SLB. AFM experiments showed that incubation with NLPs resulted in the formation of topographical features with similar size distribution as that of defects observed in control samples. Although the structural rearrangement was too complex to outline a clear mechanism, it verified interactions between NLPs and the SLB, which resulted in changes to the structure of the SLB as well as direct transfer of cargo from the NLP to the SLB. Lastly, preliminary FM experiments with NLPs containing embedded receptor tyrosine kinase CLIP-ErbB2/HER2 demonstrated that protein cargoes could be delivered from NLPs to PAA-cushioned SLBs.

There are notable advantages in using model membrane systems - NLPs enable access to a growing selection of membrane proteins, while SLBs create opportunities to study them in modular, two-dimensional environments. Indeed, the integration of cell-free expression into NLPs is a growing area of focus and eliminates challenging purification steps of traditional cell-based protein expression. The SLB platform is also very flexible. The composition of the SLB can also be readily adjusted to enhance or decrease loading to suit specific applications, as well as investigate the impact of membrane composition on protein function in a controlled fashion. Nevertheless, there are limitations to this approach. Namely, aging effects necessitate that experiments be conducted within a short period of time. NLPs should be utilized within 1 week of synthesis, as DLS experiments revealed that they are prone to aggregation over time (unpublished data). Likewise, SLBs are susceptible to long term lipid solubilization and should be utilized within $24 \mathrm{hr}$ or kept in lipid-saturated solution conditions to prevent equilibration induced desorption. Solution conditions must also be considered carefully. While we did not closely examine NLP stability under varied ionic strength conditions, we found that NLPs tended to aggregate in deionized water and therefore recommend that they be stored in solutions with sufficiently high ionic strength (> $100 \mathrm{mM}$ ). In general, NLP aggregates can be dispersed by quick sonication, vortexing, or rapid pipetting.

In conclusion, this work shows that NLP-mediated transfer can be used to introduce lipids and membrane proteins into SLBs under well-defined conditions. Next steps should focus on transfer of different membrane proteins to SLBs, with special attention paid to target membrane composition to maximize loading. Equally important will be verification of the structural fidelity and functionality of ErbB2/HER2 after delivery, likely through observation of domain recognition and binding by fluorescently labeled antibodies.

\section{Supplementary Material}

Refer to Web version on PubMed Central for supplementary material. 


\section{ACKNOWLEDGEMENT}

AFM images were recorded at the NEAT ORU Keck Spectral Instrument Facility. We thank Prof. Marjorie Longo and Prof. Tina Jeoh at the University of California, Davis for providing access to the instruments used to characterize NLPs.

Funding Sources

This work was supported by the National Science Foundation Chemistry Division through grant CHE-1413745, the University of California, Davis Biotechnology Training Program Fellowship, and the National Institutes of Health under award numbers R21AI120925, R01CA155642 and R01GM117342. Work was also performed under the auspices of the U.S. Department of Energy by Lawrence Livermore National Laboratory under Contract DEAC52-07NA27344.

\section{REFERENCES}

1. Jonas A; Kezdy KE; Wald JH, Defined apolipoprotein A-I conformations in reconstituted high density lipoprotein discs. The Journal of biological chemistry 1989, 264 (9), 4818-24. [PubMed: 2494178]

2. Fischer NO; Weilhammer DR; Dunkle A; Thomas C; Hwang M; Corzett M; Lychak C; Mayer W; Urbin S; Collette N; Chiun Chang J; Loots GG; Rasley A; Blanchette CD, Evaluation of Nanolipoprotein Particles (NLPs) as an In Vivo Delivery Platform. PLoS ONE 2014, 9 (3), e93342. [PubMed: 24675794]

3. Numata M; Grinkova YV; Mitchell JR; Chu HW; Sligar SG; Voelker DR, Nanodiscs as a therapeutic delivery agent: inhibition of respiratory syncytial virus infection in the lung. International journal of nanomedicine 2013, 8, 1417-1427. [PubMed: 23717040]

4. Cappuccio JA; Blanchette CD; Sulchek TA; Arroyo ES; Kralj JM; Hinz AK; Kuhn EA; Chromy BA; Segelke BW; Rothschild KJ; Fletcher JE; Katzen F; Peterson TC; Kudlicki WA; Bench G; Hoeprich PD; Coleman MA, Cell-free co-expression of functional membrane proteins and apolipoprotein, forming soluble nanolipoprotein particles. Molecular \& cellular proteomics : MCP 2008, 7 (11), 2246-53. [PubMed: 18603642]

5. He W; Scharadin TM; Saldana M; Gellner C; Hoang-Phou S; Takanishi C; Hura GL; Tainer JA; Carraway Iii KL; Henderson PT; Coleman MA, Cell-free expression of functional receptor tyrosine kinases. Scientific Reports 2015, 5, 12896. [PubMed: 26274523]

6. Leitz AJ; Bayburt TH; Barnakov AN; Springer BA; Sligar SG, Functional reconstitution of beta(2)adrenergic receptors utilizing self-assembling Nanodisc technology. Biotechniques 2006, 40 (5), 601-+. [PubMed: 16708760]

7. Gilmore SF; Blanchette CD; Scharadin TM; Hura GL; Rasley A; Corzett M; Pan C.-x.; Fischer NO; Henderson PT, Lipid Cross-Linking of Nanolipoprotein Particles Substantially Enhances Serum Stability and Cellular Uptake. ACS applied materials \& interfaces 2016, 8 (32), 20549-20557. [PubMed: 27411034]

8. Plochberger B; Röhrl C; Preiner J; Rankl C; Brameshuber M; Madl J; Bittman R; Ros R; Sezgin E; Eggeling C; Hinterdorfer P; Stangl H; Schütz GJ, HDL particles incorporate into lipid bilayers - a combined AFM and single molecule fluorescence microscopy study. Scientific Reports 2017, 7 (1), 15886. [PubMed: 29162870]

9. Rothblat GH; Phillips MC, High-density lipoprotein heterogeneity and function in reverse cholesterol transport. Curr Opin Lipidol 2010, 21.

10. Lund-Katz S; Phillips MC, High density lipoprotein structure-function and role in reverse cholesterol transport. Sub-cellular biochemistry 2010, 51, 183-227. [PubMed: 20213545]

11. Williams DL; de la Llera-Moya M; Thuahnai ST; Lund-Katz S; Connelly MA; Azhar S; Anantharamaiah GM; Phillips MC, Binding and Cross-linking Studies Show That Scavenger Receptor BI Interacts with Multiple Sites in Apolipoprotein A-I and Identify the Class A Amphipathic a-Helix as a Recognition Motif. Journal of Biological Chemistry 2000, 275 (25), 18897-18904. [PubMed: 10858447]

12. Rothblat GH; Phillips MC, High-density lipoprotein heterogeneity and function in reverse cholesterol transport. Current opinion in lipidology 2010, 21 (3), 229-238. [PubMed: 20480549] 
13. Chromy BA; Arroyo E; Blanchette CD; Bench G; Benner H; Cappuccio JA; Coleman MA; Henderson PT; Hinz AK; Kuhn EA; Pesavento JB; Segelke BW; Sulchek TA; Tarasow T; Walsworth VL; Hoeprich PD, Different apolipoproteins impact nanolipoprotein particle formation. J Am Chem Soc 2007, 129 (46), 14348-54. [PubMed: 17963384]

14. Cleveland TE; He W; Evans AC; Fischer NO; Lau EY; Coleman MA; Butler P, Small-angle X-ray and neutron scattering demonstrates that cell-free expression produces properly formed discshaped nanolipoprotein particles. Protein Science 2018, 27 (3), 780-789. [PubMed: 29266475]

15. Blanchette CD; Cappuccio JA; Kuhn EA; Segelke BW; Benner WH; Chromy BA; Coleman MA; Bench G; Hoeprich PD; Sulchek TA, Atomic force microscopy differentiates discrete size distributions between membrane protein containing and empty nanolipoprotein particles. Biochimica Et Biophysica Acta-Biomembranes 2009, 1788 (3), 724-731.

16. Bayburt TH; Grinkova YV; Sligar SG, Self-Assembly of Discoidal Phospholipid Bilayer Nanoparticles with Membrane Scaffold Proteins. Nano Letters 2002, 2 (8), 853-856.

17. Bricarello DA; Smilowitz JT; Zivkovic AM; German JB; Parikh AN, Reconstituted lipoprotein: a versatile class of biologically-inspired nanostructures. ACS Nano 2011, 5 (1), 42-57. [PubMed: 21182259]

18. Gao TJ; Petrlova J; He W; Huser T; Kudlick W; Voss J; Coleman MA, Characterization of De Novo Synthesized GPCRs Supported in Nanolipoprotein Discs. Plos One 2012, 7 (9).

19. Rawson S; Davies S; Lippiat JD; Muench SP, The changing landscape of membrane protein structural biology through developments in electron microscopy. Molecular membrane biology 2016, 33 (1-2), 12-22. [PubMed: 27608730]

20. Yin H; Flynn AD, Drugging Membrane Protein Interactions. Annual review of biomedical engineering 2016, 18 (1), 51-76.

21. White S Membrane Proteins of Known 3D Structure. http://blanco.biomol.uci.edu/mpstruc/ (accessed 21 January).

22. Ghosh E; Kumari P; Jaiman D; Shukla AK, Methodological advances: the unsung heroes of the GPCR structural revolution. Nature Reviews Molecular Cell Biology 2015, 16, 69. [PubMed: 25589408]

23. Dawaliby R; Trubbia C; Delporte C; Masureel M; Van Antwerpen P; Kobilka BK; Govaerts C, Allosteric regulation of $\mathrm{G}$ protein-coupled receptor activity by phospholipids. Nature Chemical Biology 2015, 12, 35. [PubMed: 26571351]

24. Saliba A-E; Vonkova I; Gavin A-C, The systematic analysis of protein-lipid interactions comes of age. Nature Reviews Molecular Cell Biology 2015, 16, 753. [PubMed: 26507169]

25. Scharadin TM; Saldana M; Schlein M; Hoang-Phou S; Trans D; Chang D; He W; Lam K; Carraway KL; Coleman MA; Henderson PT, Using NLPs to study EGFR structure, activation, and inhibition. Cancer Research 2014, 74 (19).

26. Scharadin TM; He W; Yiannakou Y; Tomilov AA; Saldana M; Cortopassi GA; Carraway KL 3rd; Coleman MA; Henderson PT, Synthesis and biochemical characterization of EGF receptor in a water-soluble membrane model system. PloS one 2017, 12 (6), e0177761-e0177761. [PubMed: 28586369]

27. Lai G; Renthal R, Integral Membrane Protein Fragment Recombination after Transfer from Nanolipoprotein Particles to Bicelles. Biochemistry 2013, 52 (52), 9405-9412. [PubMed: 24328096]

28. Gao TJ; Blanchette CD; He W; Bourguet F; Ly S; Katzen F; Kudlicki WA; Henderson PT; Laurence TA; Huser T; Coleman MA, Characterizing diffusion dynamics of a membrane protein associated with nanolipoproteins using fluorescence correlation spectroscopy. Protein Science 2011, 20 (2), 437-447. [PubMed: 21280134]

29. Watkins EB; Kuhl TL; Majewski J; Miller CE; Johannes L; Gao H; Dennison AJ, Glycolipid clustering and lipid reorganization induced by bacterial toxin binding to model membranes. European Biophysics Journal with Biophysics Letters 2013, 42, S157-S157.

30. Li E; Merzlyakov M; Lin J; Searson P; Hristova K, Utility of surface-supported bilayers in studies of transmembrane helix dimerization. Journal of Structural Biology 2009, 168 (1), 53-60. [PubMed: 19303932] 
31. Sezgin E; Schwille P, Model membrane platforms to study protein-membrane interactions. Molecular membrane biology 2012, 29 (5), 144-54. [PubMed: 22831167]

32. Kurniawan J; Ventrici de Souza JF; Dang AT; Liu G.; Kuhl TL, Preparation and Characterization of Solid-Supported Lipid Bilayers Formed by Langmuir-Blodgett Deposition: A Tutorial. Langmuir 2018, 34 (51), 15622-15639. [PubMed: 30465730]

33. Sezgin E; Levental I; Mayor S; Eggeling C, The mystery of membrane organization: composition, regulation and roles of lipid rafts. Nature Reviews Molecular Cell Biology 2017, 18, 361. [PubMed: 28356571]

34. El-khouri RJ; Bricarello DA; Watkins EB; Kim CY; Miller CE; Patten TE; Parikh AN; Kuhl TL, pH Responsive Polymer Cushions for Probing Membrane Environment Interactions. Nano Letters 2011, 11 (5), 2169-2172. [PubMed: 21500840]

35. Watkins E; El-khouri RJ; Miller C; Seaby BG; Majewski J; Marques CM; Kuhl T, Structure and thermodynamics of lipid bilayers on polyethylene glycol cushions: Fact and fiction of PEG cushioned membranes. Langmuir 2011.

36. Pace H; Simonsson Nyström L; Gunnarsson A; Eck E; Monson C; Geschwindner S; Snijder A; Höök F, Preserved Transmembrane Protein Mobility in Polymer-Supported Lipid Bilayers Derived from Cell Membranes. Analytical chemistry 2015, 87 (18), 9194-9203. [PubMed: 26268463]

37. Nair PM; Salaita K; Petit RS; Groves JT, Using patterned supported lipid membranes to investigate the role of receptor organization in intercellular signaling. Nat. Protoc 2011, 6, 523. [PubMed: 21455188]

38. Lai G; Forti KM; Renthal R, Kinetics of lipid mixing between bicelles and nanolipoprotein particles. Biophysical chemistry 2015, 197, 47-52. [PubMed: 25660392]

39. Zeno WF; Rystov A; Sasaki DY; Rishud SH; Longo ML, Crowding-Induced Mixing Behavior of Lipid Bilayers: Examination of Mixing Energy, Phase, Packing Geometry, and Reversibility. Langmuir 2016, 32 (18), 4688-4697. [PubMed: 27096947]

40. Patriarchi T; Shen A; He W; Baikoghli M; Cheng RH; Xiang YK; Coleman MA; Tian L, Nanodelivery of a functional membrane receptor to manipulate cellular phenotype. Scientific Reports 2018, 8 (1), 3556. [PubMed: 29476125]

41. Kurniawan J; Kuhl TL, Characterization of solid-supported dipalmitoylphosphatidylcholine membranes containing cholesterol. Langmuir 2015, 31 (8), 2527-32. [PubMed: 25658965]

42. Zeno WF; Hilt S; Aravagiri KK; Risbud SH; Voss JC; Parikh AN; Longo ML, Analysis of lipid phase behavior and protein conformational changes in nanolipoprotein particles upon entrapment in sol-gel-derived silica. Langmuir 2014, 30 (32), 9780-8. [PubMed: 25062385]

43. Kienle DF; de Souza JV; Watkins EB; Kuhl TL, Thickness and refractive index of DPPC and DPPE monolayers by multiple-beam interferometry. Analytical and bioanalytical chemistry 2014, 406 (19), 4725-33. [PubMed: 24842403]

44. Shen W-J; Azhar S; Kraemer FB, SR-B1: A Unique Multifunctional Receptor for Cholesterol Influx and Efflux. Annual review of physiology 2018, 80, 95-116.

45. Plochberger B; Axmann M; Röhrl C; Weghuber J; Brameshuber M; Rossboth BK; Mayr S; Ros R; Bittman R; Stangl H; Schütz GJ, Direct observation of cargo transfer from HDL particles to the plasma membrane. Atherosclerosis 2018, 277, 53-59. [PubMed: 30173079]

46. Arnulphi C; Sánchez SA; Tricerri MA; Gratton E; Jonas A, Interaction of human apolipoprotein AI with model membranes exhibiting lipid domains. Biophysical journal 2005, 89 (1), 285-295. [PubMed: 15849246] 


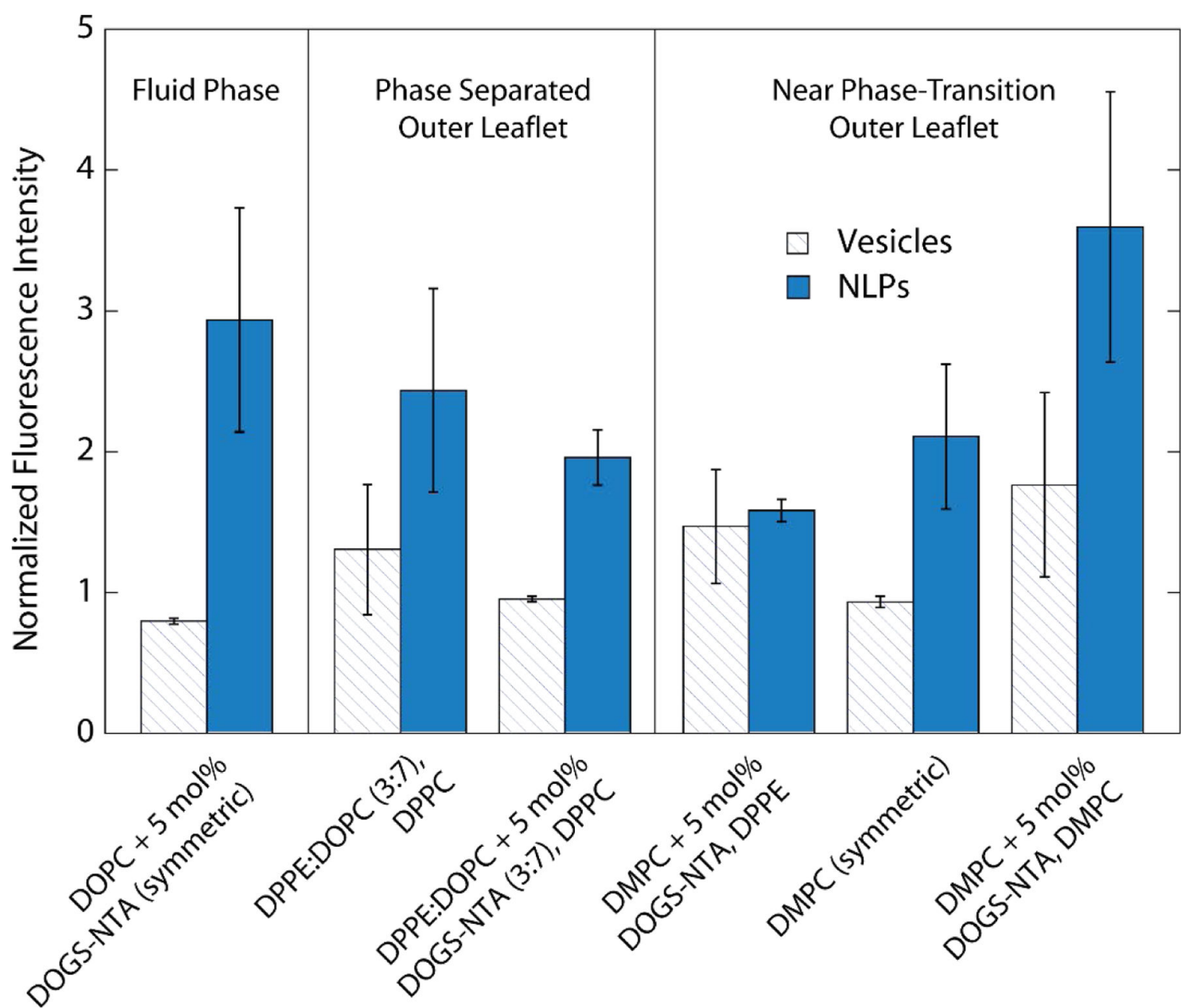

\section{SLB Composition [Outer Leaflet, Inner Leaflet]}

Figure 1.

Degree of lipid transfer as a function of composition, as indicated by the mean fluorescence intensity of SLBs after incubation with MSP1D1-NLPs and vesicles containing $2 \mathrm{~mol} \%$ Rhodamine-DHPE at room temperature, normalized by the fluorescence intensity of unlabeled SLBs on glass. Transfer was enhanced by the presence of bilayer defects, which are abundant in fluid phase (DOPC) membranes and in membranes not coupled to a gel phase (DPPC or DPPE) inner leaflet. For near phase-transition (DMPC) membranes, transfer was further enhanced by addition of DOGS-NTA, a nickel-chelating lipid capable of specific binding to NLPs via the polyhistidine-tag incorporated in each apolipoprotein sequence. 

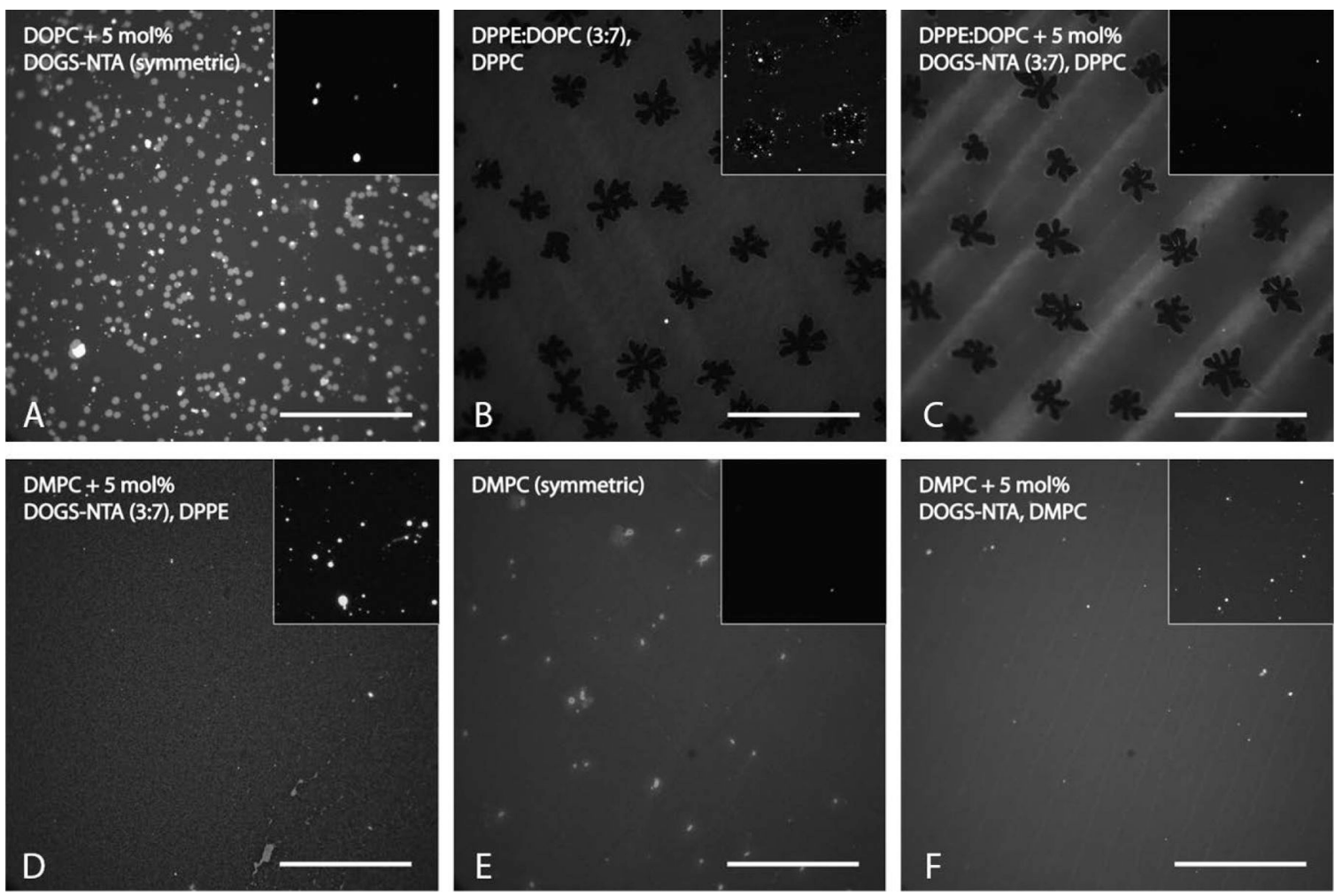

Figure 2.

FM images of different composition (label: outer leaflet, inner leaflet) SLBs after incubation with MSP1D1-NLPs and vesicles (insets) containing fluorescent lipid Rhodamine B-DHPE (scale bar $=100 \mu \mathrm{m}$ for image and inset) at room temperature. (A) Introduction of DMPC from NLPs into fluid phase DOPC SLBs resulted in phase separation and partitioning of the fluorophore into liquid-disordered $\left(\mathrm{L}_{\mathrm{o}}\right)$ domains. $(\mathrm{B}, \mathrm{C})$ In phase-separated DPPE:DOPC SLBs, gel-phase domains appeared dark in contrast with the surrounding fluid-phase matrix. (D) Lipid transfer was suppressed in DMPC SLBs coupled to a gel-phase inner leaflet despite having polyhistidine-binding DOGS-NTA. Exchange in DMPC membranes was enhanced by (E) changing the inner leaflet to DMPC, (F) and then incorporating DOGSNTA. Intensity was scaled identically for all images shown here. 

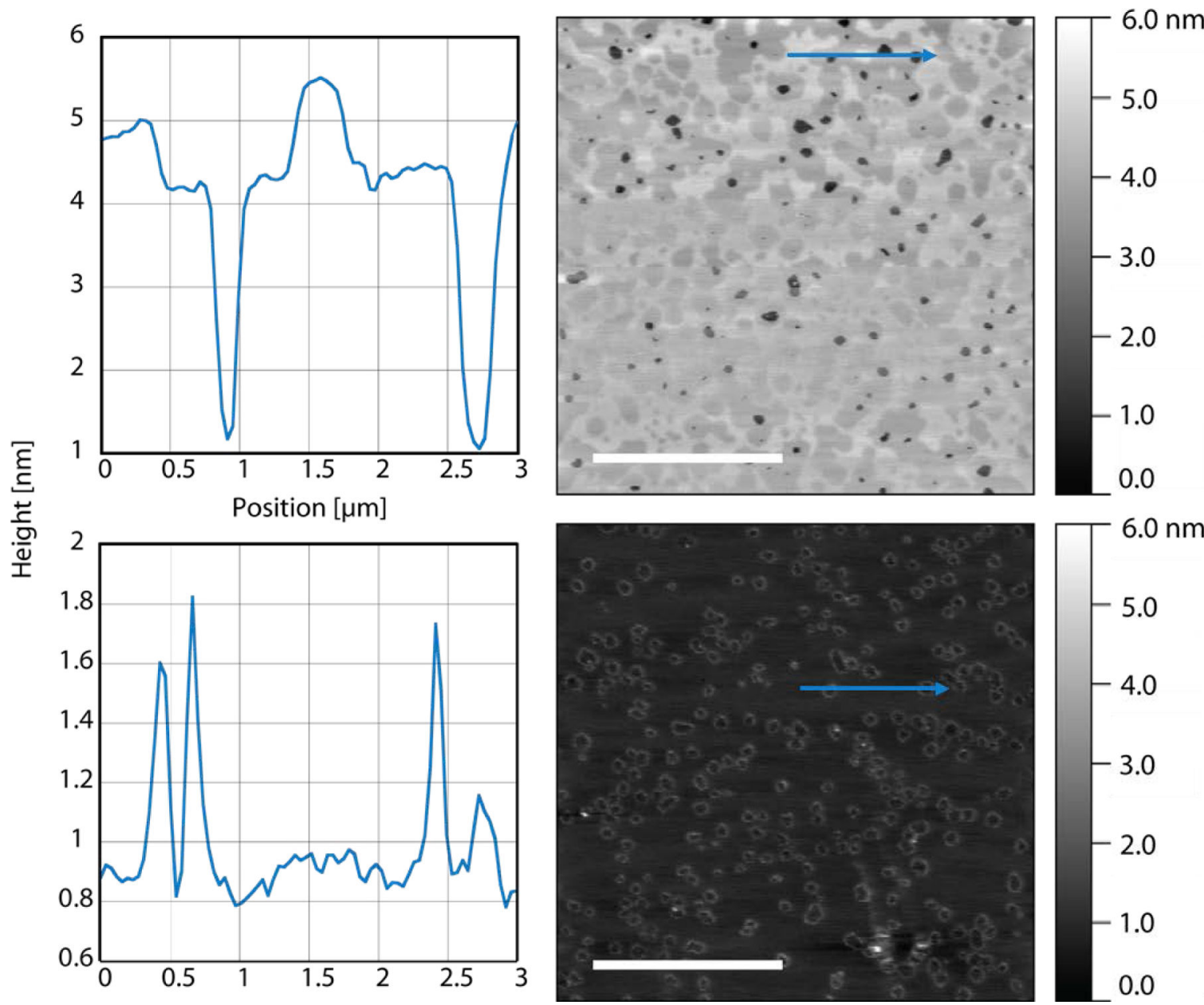

Figure 3.

(Right) AFM topographic images (scale bar $=3 \mu \mathrm{m}$ ) and (Left) line profile scans of DMPC + 5 mol\% DOGS-NTA (inner leaflet DPPE) SLBs recorded at room temperature in PBS buffer, after $12 \mathrm{hr}$ incubation and subsequent rinsing. (Top) SLBs incubated without NLPs exhibited defects in the form of holes with an average depth of $\sim 5 \mathrm{~nm}$ due to solubilization of lipid over time. Since SLBs were imaged under ambient conditions near the phase transition temperature of DMPC $\left(\mathrm{T}_{\mathrm{m}}=24^{\circ} \mathrm{C}\right)$, phase separation was observed. (Bottom) SLBs incubated with NLPs presented raised ring-like features. Line profile scans revealed that holes were not present, suggesting that defects had become filled as a result of materials transfer from NLPs. 

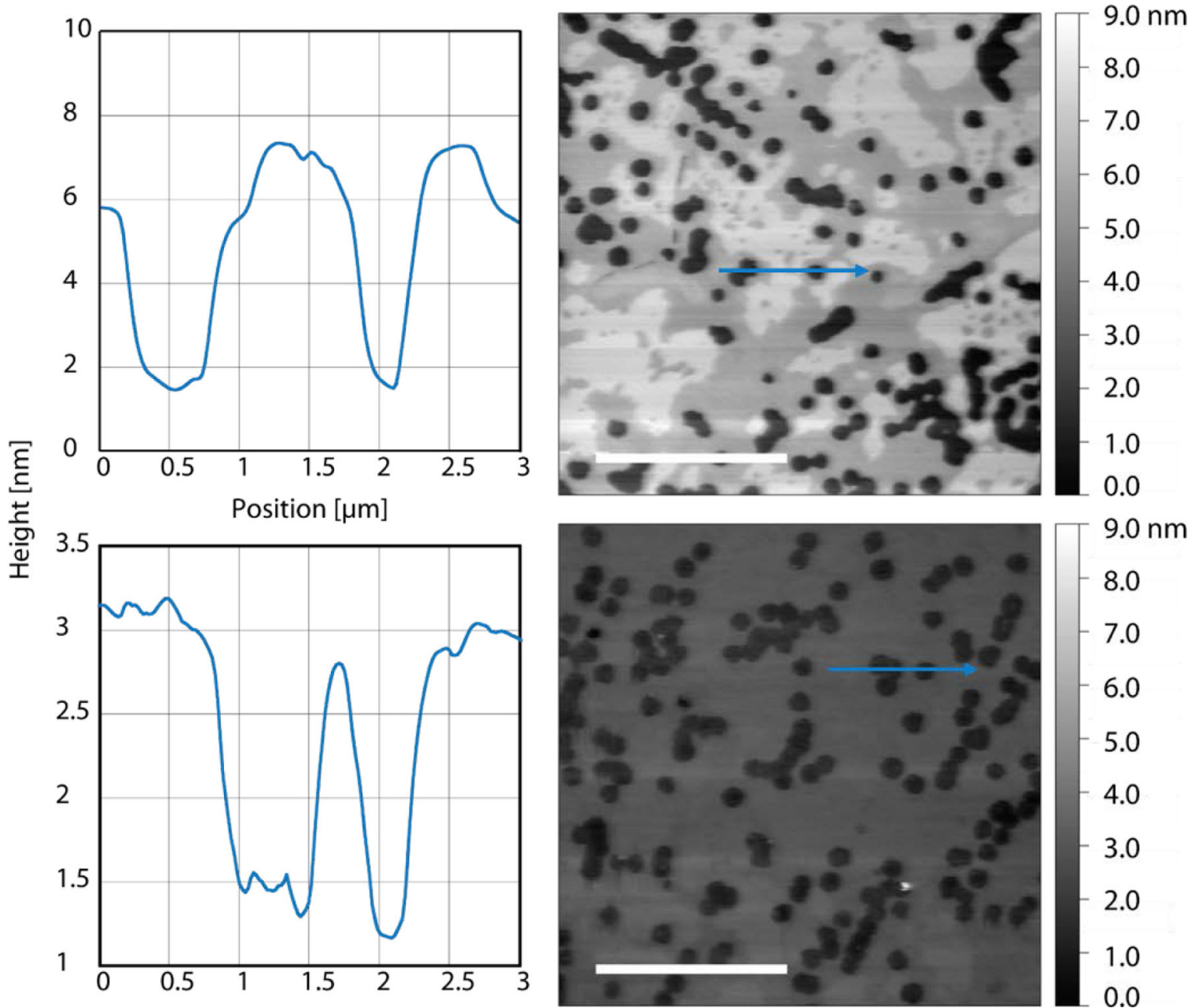

Figure 4.

(Right) AFM topographic images (scale bar $=3 \mu \mathrm{m}$ ) and (Left) line profile scans of DPPC:DOPC + 5 mol\% DOGS-NTA:Chol (9:9:2) (inner leaflet DPPC) SLBs recorded at room temperature in PBS buffer, after $12 \mathrm{hr}$ incubation and subsequent rinsing. (Top) SLBs incubated without NLPs exhibited phase separation and defects in the form of holes with an average depth of $\sim 5 \mathrm{~nm}$. (Bottom) Phase separation was suppressed in SLBs incubated with NLPs. Further, defects appeared partially filled with an average depth of $\sim 1.5 \mathrm{~nm}$. 


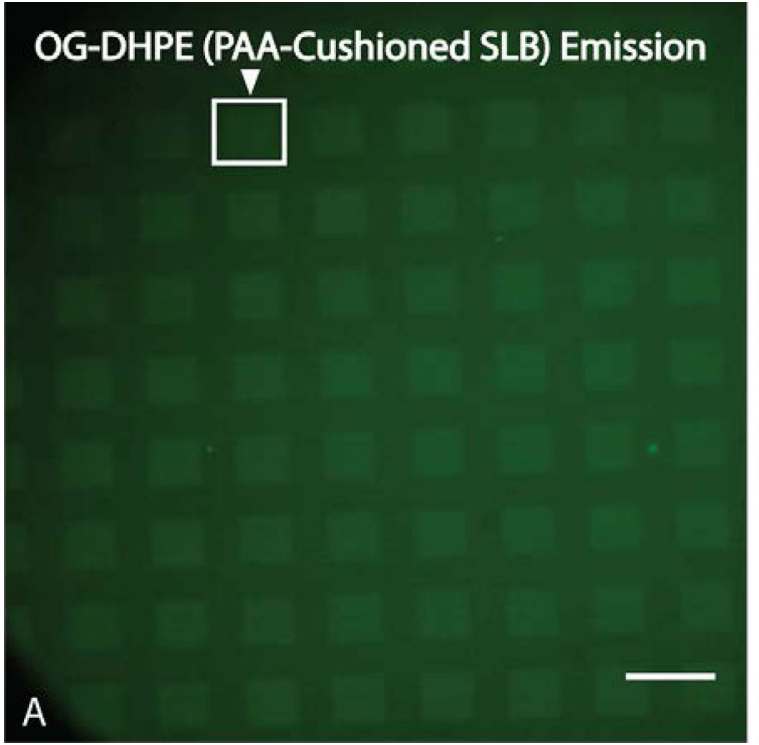

TR-DHPE (NLP Cargo) Emission

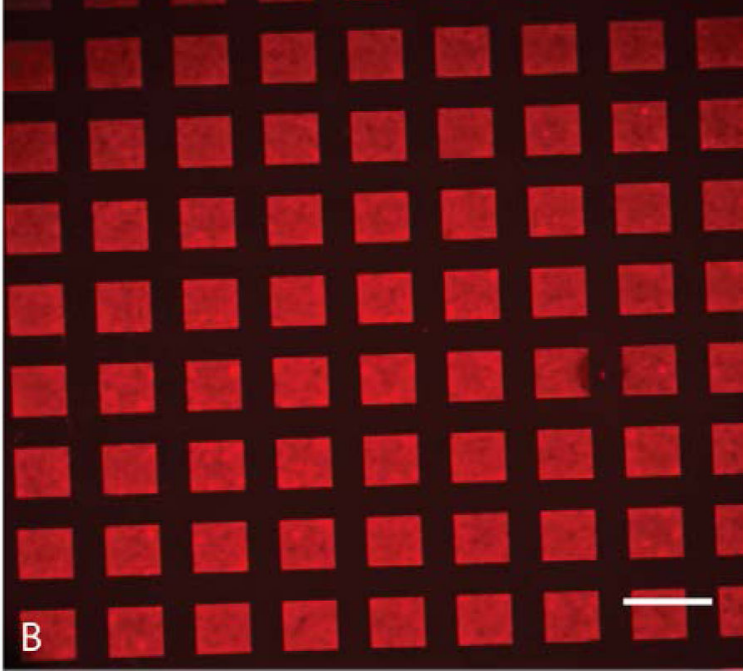

Figure 5.

FM of an OG-DHPE-labeled micropatterned PAA-cushioned (DMPC + 5 mol\% DOGSNTA) SLB after incubation with NLPs containing TR-DHPE (scale bar $=200 \mu \mathrm{m})$. Lipid transfer was evidenced by the co-localization of fluorescent emission from (A) OG-DHPE and (B) TR-DHPE. 

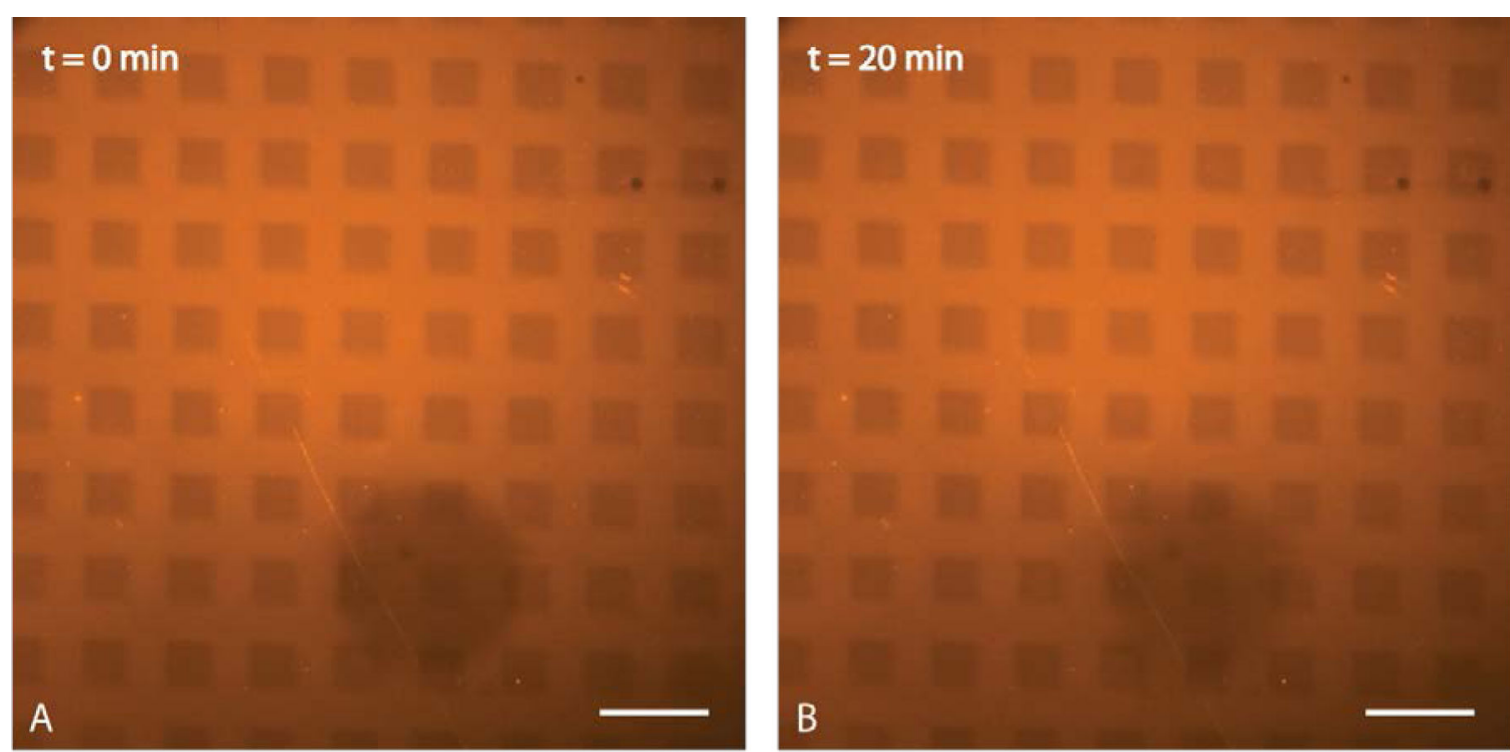

Figure 6.

FRAP of a micropatterned PAA-cushioned (DMPC + 5 mol\% DOGS-NTA) SLB after incubation with CLIP-ErbB2-NLPs (scale bar $=200 \mu \mathrm{m}$ ). Because the bilayer was deposited after patterning and passivation of exposed surfaces, parts of the membrane "bridged" across PAA patches (squares) to form a continuous bilayer across the surface. (A) Photobleaching a small region of the SLB (darkened circular area, diameter $\sim 150 \mu \mathrm{m}$ ) verified that CLIPErbB2/HER2 incorporated into both directly supported and suspended areas of the bilayer. (B) Partial recovery during FRAP, evidenced by brightening and the blurring of edges in the photobleached region, was observed in suspended regions over time. 